\title{
Diseñando un simulador de ecosistemas. Una experiencia STEM de enseñanza de dinámica de los ecosistemas, funciones matemáticas y programación
}

\author{
Jordi Domènech-Casal (iD \\ Departament de Didàctica de la Matemàtica i les Ciències Experimentals, Universitat Autònoma de \\ Barcelona, Cerdanyola. Institut de Secundària Marta Estrada, Granollers. Programa STEAMCat del \\ Departament d'Educació de la Generalitat de Catalunya, España.jdomen44@xtec.cathttps
}

[Recibido: 3 Agosto 2019. Revisado: 27 Diciembre 2019. Aceptado: 11 Abril 2020]

\begin{abstract}
Resumen: El aprendizaje sobre los ecosistemas y su carácter dinámico e interrelacionado es un aspecto clave para la enseñanza de la Ecología. El alumnado tiene dificultades para desarrollar una visión interdependiente de los elementos que constituyen un ecosistema. El enfoque STEM se ha propuesto como vía para un aprendizaje más integrado y suele proponerse en la forma de Aprendizaje Basado en Proyectos interdisciplinarios, también como vía para superar sesgos de género en el aprendizaje de las ciencias. Se ha desarrollado y aplicado un proyecto STEM basado en la programación de un simulador de ecosistemas con distintas funciones matemáticas por parte de alumnado de $4^{\circ}$ de ESO. Se describen los resultados de su aplicación y se analizan aspectos como la utilidad de la interdisciplinariedad y el desarrollo de habilidades científicas y tecnológicas.
\end{abstract}

Palabras clave: STEM, Aprendizaje Basado en Proyectos, modelización, interdisciplinar, ecosistemas.

Designing an ecosystem simulator. Teaching ecosystem dynamics, mathematical functions and programming in a STEM experience.

Abstract: Learning on ecosystems and their dynamism and interelation is a key aspect on Ecology teaching. Students have difficulties to develop an interdependent view of elements constituting an ecosystem. STEM has been proposed as a perspective to a more integrated learning and is usually proposed in the shape of interdisciplinar Project-Based Learning (PBL) activities, also to overcome gender biases in Science learning. We have developed and applied a STEM PBL activity where 15-years-old students design and program an ecosystem simulator through several mathematical functions. We describe the results of the application and analyze interdisciplinarity outcomes and the development of of scientific and technologic skills

Keywords: STEM, Project-Based Learning, modelling, interdisciplinar, ecosystems

Para citar este artículo: Domènech-Casal J. (2020) Construyendo un simulador de ecosistemas. Una experiencia STEM de enseñanza de dinámica de los ecosistemas, funciones matemáticas y programación. Revista Eureka sobre Enseñanza y Divulgación de las Ciencias 17(3), 3202. doi: 10.25267/Rev_Eureka_ensen_divulg_cienc.2020.v17.i3.3202

\section{Introducción}

La enseñanza sobre los ecosistemas es un tema clave para la comprensión de problemáticas socioambientales y la toma de decisiones participadas por la ciencia (Vilches y Gil 2007). La crisis hídrica, las problemáticas por disminución de polinizadores, el calentamiento global, la los microplásticos, la acidificación oceánica son sólo algunos ejemplos de retos que deberemos asumir como especie, desde la política y la participación ciudadana, que requieren una comprensión profunda de los elementos que configuran el ecosistema y de las relaciones dinámicas que se establecen entre ellos.

En el aprendizaje sobre ecosistemas el alumnado suele presentar dificultades en dos aspectos: por un lado, en integrar en un mismo sistema lo "vivo" y lo "no vivo" y relacionar los componentes abióticos (White, 2000, Mohan, Chen y Anderson 2009, Sánchez y Pontes 2010, Jaén, Esteve y Baños 2018). En particular, el alumnado no identifica la interacción entre bioma y biotopo (Shepardson, Wee, Priddy y Harbor 2007) ni relaciona los ciclos de materia y

\author{
Revista Eureka sobre Enseñanza y Divulgación de las Ciencias \\ Universidad de Cádir. APAC-Eureka. ISSN: 1697-011X \\ bttp://dx.doi.org/10.25267/Rev_Eureka_ensen_divulg_cienc.2020.v17.i3.3202 \\ http:/ / reuredc.uca.es
}


energía entre sí (Correa 2013). Igualmente, el alumnado tiene problemas para elaborar explicaciones de esos ciclos en base a los procesos de producción y consumo de alimento, como la fotosíntesis o el papel de los organismos degradadores (Leach, Driver, Scott y WoodRobinson 1996a, Grotzer y Bell-Basca 2003). Aún así, incluso cuando el alumnado consigue usar esos conceptos para interpretar los ecosistemas, la mayoría de estudiantes entienden esas interacciones exclusivamente desde el punto de vista de la alimentación y como relaciones de predación y no de cooperación (Leach, Driver, Scott y Wood-Robinson 1996b, Rojero 1999, Eilam. 2002). Esto conlleva una visión del ecosistema como una cadena alimentaria lineal y simple, en lugar de una red dinámica (Gil y Martínez 1992) y no permite desarrollar habilidades como predecir cambios e impactos en los ecosistemas (Murillo y Gil 2009). Esta visión ha sido quizás reforzada por visiones sesgadas en los libros de texto (Maldonado, González y Jiménez 2007), que ofrecen una visión mecanicista y teleológica poco sistémica en sus definiciones de términos como "red trófica" o "equilibrio".

Por otro lado, la aproximación del alumnado al concepto de ecosistema suele centrarse en lo que puede verse a simple vista sin tener en cuenta aspectos como el dinamismo o el concepto de escala (Bell-Basca, Grotzer, Donis y Shaw 2000, White 2000, Bermúdez y De Longhi 2008) o los distintos niveles tróficos en un ecosistema.

Para superar estas dificultades, distintos autores consideran que es importante que las secuencias de aprendizaje ayuden a comprender el carácter multifactorial e interdependiente de las dinámicas ecológicas (Jaén et al 2018). Recomiendan que esto se haga integrando de forma sucesiva los distintos elementos de los conceptos de ecosistema en forma de modelos progresivamente más complejos desde la identificación de elementos, al papel de las relaciones, hasta la interdependencia en red (García 1997), preferiblemente en el marco de la resolución de problemas (García 2003, Cano-Velásquez, Zapata-Grajales, Montoya-Osorio, Villa-Ochoa 2018). En este sentido, las dinámicas propias de los ecosistemas pueden modelizarse matemáticamente y en algunos niveles educativos se usa esa posibilidad como elemento problematizador, permitiendo trabajar aspectos y parámetros como la biodiversidad, competitividad de especies, valor de biomasa o los modelos de dinámica de poblaciones exponencial o logístico (Cano-Velásquez et al 2018). También es de interés el modelo depredador-presa de Lotka-Volterra, por el cual la población de predador y presa establecen ciclos interrelacionados oscilantes (el aumento de presas conlleva un aumento de predadores, que a continuación inicia una disminución de presas y la consiguiente disminución de predadores) (Smith y Smith 2001). Estas aproximaciones permiten reforzar la idea de dinamismo e interrelación citadas anteriormente, y pueden plasmarse en fórmulas matemáticas que modelizan el comportamiento de las poblaciones en base a distintos parámetros ecológicos (Margalef 1981), como $\mathrm{N}$ (número de individuos), $\mathrm{r}$ (tasa de crecimiento), $\mathrm{t}$ (número de generaciones), $\mathrm{K}$ (total de individuos de una especie que un ecosistema es capaz de soportar), $\delta$ (impacto de predador en presas) y $\gamma$ (impacto de presas en predador):

Crecimiento poblacional exponencial $\quad \mathrm{N}_{\mathrm{t}+1}=\mathrm{N}_{\mathrm{t}}+\mathrm{r} \mathrm{N}_{\mathrm{t}}$

Crecimiento poblacional logístico $\mathrm{N}_{\mathrm{t}+1}=\mathrm{N}_{\mathrm{t}}+\mathrm{r} \mathrm{N}_{\mathrm{t}}\left[\left(\mathrm{K}-\mathrm{N}_{\mathrm{t}}\right) / \mathrm{K}\right]$

Crecimiento poblacional en sistema de predación con dos especies $\mathrm{X}$ e $\mathrm{Y}$

$$
\begin{aligned}
& \mathrm{Xt}+1=\mathrm{Xt}+\mathrm{rXt}-\delta \mathrm{Xt} \mathrm{Yt} \\
& \mathrm{Yt}+1=\mathrm{Yt}+\gamma \mathrm{Xt} \mathrm{Yt}-\mathrm{c} \mathrm{Yt}
\end{aligned}
$$

Algunos autores plantean el trabajo con este tipo de aproximaciones como vía de aprendizaje de la modelización matemática (Blomhǿj 2004), un aspecto interdisciplinar de interés por razones que comentamos en el siguiente apartado. 


\section{La perspectiva STEM y el Aprendizaje Basado en Proyectos}

En los últimos años ha emergido con fuerza el acrónimo STEM como enfoque para la enseñanza de las ciencias. Las siglas STEM se corresponden en inglés a los ámbitos de Ciencia, Tecnología, Ingeniería y Matemáticas (Couso 2017) y son cada vez más presentes en los ámbitos de innovación educativa. El origen de estas siglas no es didáctico, sino político (Domènech-Casal 2018), y se enmarca en la necesidad del mundo industrial y económico desarrollado de responder a un reto "logístico". El informe Rocard (Rocard et al 2007) describió una caída en las vocaciones científico-tecnológicas, especialmente acentuada en relación al género y el origen socio-económico: las alumnas en general y el alumnado de familias de bajo nivel socio-económico accede en muy baja proporción a las vocaciones científico-tecnológicas (lo que supone una pérdida de talento y diversidad de perspectivas para este campo). Mientras que la caída de las vocaciones se atribuía al uso de metodologías poco atractivas y poco competenciales (lo que llevó al reciente auge de financiación europea de innovación en enseñanza mediante la Indagación), el sesgo de género se ha atribuido a visiones de género estereotipadas. Éste último aspecto ha sido corroborado por los datos: mientras las alumnas subestiman sus propias capacidades en estos ámbitos, los alumnos (en masculino) se sobreestiman (Couso 2017, Sainz, Castaño, Meneses, Fàbregues, Müller, Rodó, Martínez, Romano, Arroyo y Garrido 2017, UNESCO 2017). A esto hay que añadir roles de género por los que -incluso dentro del ámbito científico y tecnológico- las alumnas son dirigidas (por mensajes sociales de los media, las familias, o el propio profesorado) a profesiones de "cuidados" (medicina, medio ambiente) mientras que ellos lo son a profesiones de "ingeniería" (industriales y tecnológicas), masculinizadas por estereotipos.

Igualmente, el entramado empresarial y tecnológico ha detectado las dificultades de transferencia industrial de innovaciones como los transgénicos o la nanotecnología, debido a un rechazo de la población, que se atribuye en muchos casos a falta de formación científica. Como reacción a este escenario complejo, distintos países desarrollados (como EEUU, la UE, China o Japón) han iniciado campañas en distintos ámbitos (empresarial, jurídico,...y educativo), con un triple objetivo político en respuesta a ese conjunto, que podríamos resumir en: 1) promover las vocaciones y la formación para profesiones científico-tecnológicas, 2) eliminar el sesgo de género y socioeconómico en estas vocaciones, 3) desarrollar la competencia científica y tecnológica para la ciudadanía. (Domènech-Casal, 2019a)

La proyección de este triple objetivo político sobre el mundo educativo ha resultado en las siglas STEM, que agrupan a su alrededor distintos esfuerzos para hacer aportaciones a esos tres objetivos políticos. Por decirlo de algún modo, STEM no es una metodología, sino todo lo que contribuye a avanzar en los objetivos políticos STEM (metodologías, logísticas, proridades curriculares, enfoques de género...) (Domènech-Casal, 2019a). Esto hace que en la práctica el término STEM esté asociado a múltiples significados (Metodologías, robótica, programación, perspectiva de género y tecnología, ciudadanía...) (Brown, Brown, Reardon y Merrill 2011, Bybee 2010, Martín-Páez, Aguilera, Perales-Palacios, Vilchez-González 2019, Akerson, Burgess, Gerber y Guo 2018). Además, los objetivos competenciales STEM suelen vincularse por un lado a la adquisición de competencias propias de las áreas implicadas (indagar, diseñar, modelizar) y al desarrollo de lo que se ha llamado "soft skills" o habilidades prácticas vinculadas a la autonomía, como el trabajo en equipo, el análisis crítico, la creatividad o el Pensamiento computacional (Wing 2006, Simarro y Couso 2016, Couso 2017).

Desde un punto de vista metodológico, la materialización más común del empuje STEM son actividades interdisciplinarias problematizadas que incluyan al menos dos de las áreas que configuran el acrónimo (Ciencia, Tecnología, Ingeniería y Matemáticas,..) -notar que en estas propuestas el término "Ingeniería" ha acabado a menudo interpretándose más como un 
contexto de acción que como un ámbito de conocimiento-. En lo referente a la didáctica de las ciencias, los objetivos STEM pueden asociarse con enfoques preexistentes al movimiento STEM, como el trabajo problematizado en contexto (Duranti y Goodwin 1992, Gilbert 2006, Blanco, España y Rodríguez 2012) (que acostumbra a requerir la interdisciplinariedad) y como caso particular de ese aprendizaje problematizado el Aprendizaje Basado en Proyectos (Sanmartí y Márquez 2017, Domènech-Casal 2018) un enfoque metodológico que comparte con el término "STEM" su emergencia en la innovación educativa y la multiplicidad de interpretaciones y significados.

El Aprendizaje Basado en Proyectos (ABP) fue propuesto como metodología de enseñanza inicialmente por Kilpatrick (1918) y tiene como aspecto fundamental la necesidad de un propósito compartido con el aprendiz para que se produzca un aprendizaje. Kilpatrick define el ABP como "actividades de aprendizaje con un propósito" y propone 4 tipos fundamentales de ABP, según el propósito que los anima: Elaborar un producto, Resolver un problema, Disfrutar de una experiencia estética y Obtener un conocimiento. Notar que en esta definición original el Aprendizaje Basado en Problemas (o Retos) sería -de hecho- un tipo de Aprendizaje Basado en Proyectos. La propuesta metodológica del Aprendizaje Basado en Proyectos se fundamenta pedagógicamente en la idea constructivista según la cual los aprendizajes son más profundos y transferibles cuando se realizan de manera instrumental en la resolución de un conflicto contextualizado y la necesidad de que los aprendices tengan un papel activo que ejercen en comunidad (Kilpatrick 1918). Esto implica que el diseño de las actividades ABP deben prever aspectos como la relación Contenidos-Contexto-Conflicto, el grado de apertura/estructuración de la actividad (hasta qué punto el alumnado puede tomar decisiones) y la la Interdisciplinariedad (Larmer, Mergendoller y Boss 2015, Domènech-Casal 2019b). Aunque se asume que promover la Interdisciplinariedad favorece un desarrollo de los contenidos más contextualizado, la transmisión de ese principio al diseño de proyectos no es evidente, como hemos comprobado en estudios previos de análisis y clasificación de distintos tipos de proyectos ABP STEM (Domènech-Casal, Lope y Mora, 2019).

En este artículo describimos una experiencia de aprendizaje sobre Ecología que asociamos a dos de las vías de acción STEM (la interdisciplinariedad y el desarrollo de habilidades de las áreas implicadas). Consideramos que el contexto de la Ecología como punto de partida (un contexto identificado por el alumnado como "de cuidados" y por lo tanto menos masculinizado) puede atraer al alumnado ( $y$, en especial a las alumnas) a otros aspectos matemáticos e ingenieriles, y participar en superar superar estereotipos de género. A partir de la aplicación de la actividad, hemos desarrollado los siguientes objetivos de investigación:

- Diseñar y testar una actividad ABP interdisciplinar STEM para la enseñanza sobre Ecosistemas.

- Describir si existe relación entre la autopercepción del alumnado y las habilidades medidas en Ciencia, Matemáticas y Tecnología y si ésta se ve condicionada por el género.

- Analizar aportaciones de la interdisciplinariedad al desarrollo de habilidades en las áreas STEM implicadas.

\title{
Metodología y análisis de datos
}

\author{
Diseño de la actividad
}


Se diseñó una actividad STEM ABP en la que el propósito (elaborar un producto) es construir un simulador de ecosistemas mediante el programa informático de Hoja de Cálculo Calc. Se escogió ese programa por sus posibilidades en la programación e interrelación de fórmulas y algoritmos, ser software libre y por ser un programa que el alumnado podría utilizar de nuevo fácilmente con otros propósitos con posterioridad a sus estudios. La actividad, diseñada para $4^{\circ}$ de ESO, trabaja conceptos de Ciencias (dinámicas de ecosistemas, redes tróficas, pirámides de biomasa,...), Matemáticas (funciones matemáticas, exponencial y logística, coeficientes...) y Tecnología (algoritmos, interrelación de datos de entrada y salida, secuenciación,...) y tiene como objetivo el desarrollo de habilidades para cada disciplina, que incluyen las siguientes: Programar algoritmos para el cálculo de parámetros a partir de distintas variables en Tecnología (Tec); Asociar dinámicas a funciones matemáticas para Matemáticas (Mat); Realizar predicciones del comportamiento de redes tróficas para Biología (Bio). La actividad ha sido aplicada en distintos cursos académicos e institutos (desde 2013 hasta 2019) y los materiales están disponibles para su descarga, en formato web en inglés (https://sites.google.com/a/xtec.cat/cbes/) y en formato dossier del alumno/a en catalán (https://bit.ly/2xYGo6P). Los alumnos reciben el encargo de desarrollar el simulador, y en equipos de 3 participantes siguen distintas etapas de desarrollo parcial del producto siguiendo las recomendaciones descritas en la introducción de progresar a modelos paulatinamente más complejos en el marco de una situación problematizada. A lo largo de las distintas etapas se distribuyen sesiones de introducción de contenidos, de práctica en la resolución de problemas de dinámica de poblaciones y desarrollo de habilidades, y se incorpora entre cada etapa y la siguiente un espacio de resolución de dudas y discusión en gran grupo. Para la programación del simulador, el alumnado usa las fórmulas de hoja de cálculo para, en función de unas celdas de referencia con distintos parámetros ecológicos $(\mathrm{N}, \mathrm{r}, \mathrm{K} \ldots)$, obtener datos de salida para distintas generaciones y representarlos mediante gráficos lineales y de barras. Distintos objetivos parciales del proyecto promueven el desarrollo de versiones sucesivamente más sofisticadas del simulador mediante iteración. Las distintas etapas de la actividad y los objetivos de aprendizaje se describen en la Tabla 1.

\section{Muestra y aplicación de la secuencia}

Para el análisis comprendido en este estudio, la actividad se ha aplicado durante el curso 20182019 con 18 estudiantes (8 alumnas y 10 alumnos) en la materia de Biología y Geología de 4 de ESO (Educación Secundaria Obligatoria, 15-16 años). El alumnado participante había trabajado en cursos anteriores el comportamiento matemático de las funciones exponencial y logarítmica (no la logística) y tenía nociones básicas sobre redes tróficas (no niveles tróficos) y fórmulas simples con el programa Calc. El alumnado desarrolló la actividad tal como se ha descrito en el apartado anterior y en los materiales de la actividad.

\section{Instrumentos y procedimiento de análisis}

Para testar el diseño y aplicación de la actividad se tomaron anotaciones de observaciones efectuadas durante la aplicación a lo largo de la aplicación de la actividad, y de las conversaciones durante la misma. El diseño de la actividad fue analizado en función del despliegue de distintas componentes didácticas usando la Rúbrica ABP-Map descrita en otras publicaciones (Domènech-Casal, 2018) y disponible en: https://bit.ly/2GZUARu Esta rúbrica establece para distintas componentes didácticas (Contenidos, Discurso, Conflicto, Apertura, Interdisciplinariedad) varios niveles de despliegue, de modo que permite comparar aspectos del diseño didáctico entre distintos proyectos ABP.

Tabla 1. Etapas de la actividad y objetivos de aprendizaje asociados 


\begin{tabular}{|c|c|}
\hline & cada ámbito STEM relacionado (Tec, Mat, Bio). \\
\hline $\begin{array}{l}\text { Etapa 1. Análisis de una noticia de } \\
\text { periódico sobre problemáticas ecológicas } \\
\text { en relación al krill y distintos factores } \\
\text { (bióticos y abióticos) de influencia. }\end{array}$ & $\begin{array}{l}\text { - Introducción del concepto de modelo matemático y sus } \\
\text { límites de predicción }(\text { Mat }) \\
\text { - Factor biótico y abiótico }(\text { Bio). }\end{array}$ \\
\hline $\begin{array}{l}\text { Etapas } 2 \text { y } 3 \text {. El crecimiento exponencial } \\
\text { y su modelización matemática }\end{array}$ & $\begin{array}{l}\text { - Progresiones aritméticas y geométricas. Pendiente de una } \\
\text { función y puntos de corte con los ejes. Concepto de } \\
\text { función exponencial, papel de los distintos elementos de la } \\
\text { ecuación y resolución de problemas en contexto } \\
\text { ecológico }(M a t) \text {. } \\
\text { - Tasa de crecimiento y sucesión exponencial en una } \\
\text { población en contexto ecológico }(\text { Bio }) \text {. }\end{array}$ \\
\hline $\begin{array}{l}\text { Etapa 4. Planificación. Cada equipo } \\
\text { elige un ecosistema y representa su red } \\
\text { trófica. Trabajo previo con fórmulas de } \\
\text { Calc. }\end{array}$ & $\begin{array}{l}\text { - Conceptos de red trófica y nivel trófico.(Bio). } \\
\text { - Sintaxis de programación en Calc, definición de datos de } \\
\text { salida y de entrada. Programación de fórmulas } \\
\text { interdependientes }(\mathrm{Tec}) \text {. }\end{array}$ \\
\hline $\begin{array}{l}\text { Etapas } 5 \text { y } 6 . \text { El crecimiento logístico y } \\
\text { su modelización matemática. }\end{array}$ & $\begin{array}{l}\text { - Concepto de función logística, papel de los distintos } \\
\text { elementos de la ecuación y resolución de problemas en } \\
\text { contexto ecológico }(M a t, T e c) \text {. } \\
\text { - Capacidad de carga (K) y sucesión logística en una } \\
\text { población en contexto ecológico. Políticas de protección } \\
\text { de especies (límites a la explotación, regulación) (Bio, Mat). }\end{array}$ \\
\hline $\begin{array}{l}\text { Etapa 7. Primer objetivo parcial. } \\
\text { Conseguir un simulador que reproduzca } \\
\text { el crecimiento logístico de una especie } \\
\text { para } 10 \text { generaciones. Confirmar su } \\
\text { comportamiento logístico. }\end{array}$ & $\begin{array}{l}\text { - Concepto de función logística, papel de los distintos } \\
\text { elementos de la ecuación y resolución de problemas en } \\
\text { contexto ecológico. Modelización matemática de los datos } \\
(M a t, B i o) \text {. } \\
\text { - Programación de fórmulas interdependientes y uso del } \\
\text { símbolo \$ para programar fórmulas dependientes (Mat, } \\
\text { Tec). }\end{array}$ \\
\hline $\begin{array}{l}\text { Etapas } 8 \text { y 9. Segundo objetivo parcial. } \\
\text { El simulador debe contener ahora } \\
\text { especies distintas de } 4 \text { niveles tróficos. La } \\
\text { simulación debe condicionar } r \\
\text { crecimiento a niveles de oxígeno } \\
\text { dióxido de carbono y mostrar } \\
\text { evolución de la pirámide de biomasa. }\end{array}$ & $\begin{array}{l}\text { - Red trófica y niveles tróficos. Factores bióticos y abióticos. } \\
\text { Pirámide de biomasa y energía (Bio). } \\
\text { - El papel de los coeficientes en una ecuación para } \\
\text { modificar la evolución de una función. Modelización } \\
\text { matemática de los datos }(M a t) \text {. } \\
\text { Modificación de algoritmos para reproducir } \\
\text { comportamientos de funciones y sistemas biológicos. } \\
\text { Diseño de gráficos automatizados (Tec, Mat, Bio). }\end{array}$ \\
\hline $\begin{array}{l}\text { Etapas } 10 \text { y 11. Tercer objetivo parcial. } \\
\text { La evolución de la población de las } 8 \\
\text { especies debe también considerar el } \\
\text { efecto positivo o negativo de la predación } \\
\text { entre niveles tróficos. Cada equipo debe } \\
\text { plantear además un último requerimiento } \\
\text { técnico para su simulador (incluir eventos } \\
\text { de simbiosis, disponibilidad de agua,...). }\end{array}$ & $\begin{array}{l}\text { - Relaciones competitivas. El modelo predador-presa de } \\
\text { Lotka Volterra }(B i o, M a t) \text {. } \\
\text { Concepto de ecuación diferencial. Representación gráfica } \\
\text { de la evolución interdependiente de dos variables a lo } \\
\text { largo del tiempo. El papel de los coeficientes en una } \\
\text { ecuación para modificar la evolución de una función. } \\
\text { (Mat, Bio). } \\
\text { - Simplificación de algoritmos. (Tec) }\end{array}$ \\
\hline
\end{tabular}

Para describir si existen sesgos de género en la relación entre la autopercepción del alumnado y las capacidades reales, el alumnado completó dos instrumentos: una prueba de evaluación de habilidades, y una encuesta de autopercepción. Ambos instrumentos están disponibles para su descarga en https://bit.ly/330Jp52). La prueba de evaluación de habilidades se realizó al terminar la aplicación de la actividad y comprendía tres preguntas, cada una de ellas evaluando una de las habilidades descritas en el diseño de la actividad: Programar algoritmos para el 
cálculo de parámetros a partir de distintas variables (Tecnología); Asociar dinámicas a funciones matemáticas (Matemáticas); Realizar predicciones del comportamiento de redes tróficas (Biología). Cada pregunta se calificó individualmente del 1 al 6. Las preguntas y los criterios de puntuación están disponibles junto con la prueba descargable. La encuesta de autopercepción fue completada a continuación de la prueba de evaluación de habilidades. En ella, se pidió al alumnado que valorara en una escala Likert del 1 al 6 su nivel (autopercepción) en cada una de las habilidades evaluadas en la prueba final. Se calculó para cada subgrupo (alumnas y alumnos) y cada habilidad (Tecnología, Matemáticas y Biología) la media de los resultados de la prueba y la autopercepción. Se compararon las diferencias entre alumnas y alumnos para cada parámetro (autopercepción y prueba) y ámbito (Tecnología, Matemáticas y Biología). Las diferencias entre grupos por sexos se analizaron estadísticamente. En primer lugar se testó la normalidad de las muestras mediante el test de normalidad de Shapiro-Wilk (con resultado de no normalidad) y a continuación se realizaron las correspondientes pruebas U de Mann-Whitney. Además, se calculó el sesgo de autopercepción para cada habilidad (media autopercibida - media habilidad medida en la prueba) para cada subgrupo en relación a cada una de las habilidades (Tecnología, Matemáticas, Biología).

Para analizar las aportaciones de la interdisciplinariedad al desarrollo de habilidades se realizó una encuesta de valoración entre el alumnado participante una vez terminada la actividad. Para ello se propuso al alumnado un listado de tres acciones vinculadas a los distintos ámbitos disciplinarios (Trabajar con fórmulas matemáticas, Trabajar el concepto de ecosistema, Programar un simulador) y un listado ampliado de habilidades de las materias implicadas. El alumnado valoró mediante una escala Likert $(1 \rightarrow 6)$ la aportación que habían significado las distintas acciones al desarrollo de distintas habilidades de las distintas materias implicadas. Se calculó la media de valoración para cada acción con el objetivo de detectar si según la visión del alumnado acciones de alguna disciplina hacían aportaciones al aprendizaje de habilidades de otras disciplinas.

\section{Resultados}

A lo largo de la aplicación de la actividad, se observaron distintas situaciones de interés didáctico que narramos en primer lugar para facilitar la comprensión de la actividad y ayudar a profesorado que quiera aplicar la actividad a anticipar esas situaciones. El alumnado acogió con interés el reto de construir un simulador y desarrolló sin dificultades las primeras etapas hasta superar el primer objetivo parcial de simulación de crecimiento logístico. En esa etapa, al establecer valores de entrada al azar, aparecieron curvas que presentaban anomalías en su ajuste a la curva logística esperada. En particular, el alumnado se percató que valores altos de $r$ (tasa de crecimiento) conllevaban en el simulador un comportamiento extraño: en lugar de la forma sigmoidal paradigmática transformándose plácidamente en un asíntota con la recta que representa el valor de la capacidad de carga del ecosistema (K), la población efectuaba oscilaciones alrededor de la capacidad de carga, oscilando por encima y debajo de ella (Fig. 1) . Esto generó una inesperada conversación en gran grupo sobre las causas matemáticas de ese comportamiento del simulador y su significado biológico, distintas opciones de trabajo (añadir generaciones esperando que se atenuaran las oscilaciones, aumentar la $\mathrm{K}$ esperando facilitar una "aproximación" menos brusca,...). La conversación llevó a considerar el efecto de simular de modo discreto (con intervalos) una dinámica que de hecho es continua, y el efecto en la estabilidad de los ecosistemas de tiempos de reproducción largos o muy estacionales, una idea compleja de interés desde el punto de vista matemático y biológico. 
La consecución del segundo objetivo parcial (orientado a incluir la medida de la biomasa) promovió reflexiones interesantes, relacionando el crecimiento de cada población con la biomasa total. Algunas pirámides de biomasa resultaron tener bases muy estrechas en comparación con los niveles superiores (Fig. 2). Esto implicó por un lado intentos de obtener una pirámide de biomasa "estándar" con una base amplia y pisos superiores cada vez más estrechos (en un ejercicio de inducción-deducción en el que el alumnado cambiaba valores iniciales de $\mathrm{N}, \mathrm{r}$, y la masa por individuo de cada especie, realizando ciclos rápidos de hipótesis y comprobación de impacto matemático) y por el otro una discusión sobre qué condiciones podrían hacer posible que un ecosistema se sostuviera sobre una biomasa de productores menor que la biomasa de consumidores primarios y qué riesgos entraña eso para la estabilidad del ecosistema. Sólo un equipo consiguió incorporar al crecimiento de sus poblaciones condicionantes relativos a la concentración de Oxígeno o Dióxido de Carbono.

El tercer objetivo parcial (orientado a incluir eventos de predación) fue algo más complejo, pues requirió un acompañamiento más cercano y un análisis sobre el concepto de "coeficiente" matemático. Después de una discusión de estrategias, el alumnado optó por incorporar en las fórmulas logísticas coeficientes propios de las fórmulas de Lotka-Volterra $(\gamma$ y $\delta)$ modificando con ellos las tasas de crecimiento de presas (r) y la capacidad de carga de depredadores $(\mathrm{K})$, consiguiendo incluso en algún caso efectos cíclicos parecidos a los del modelo de LotkaVolterra y desarrollando la concepción que distintos elementos pueden modificar los valores de $\mathrm{r}$ y K, como idea de interdependencia en los ecosistemas. En la conversación se puso de manifiesto que muchos alumnos consideraban sinónimos el término coeficiente y el concepto de constante física (como constante de fricción, o constante gravitacional) y se discutieron dos ideas importantes: por un lado el hecho que aunque muchas constantes son coeficientes, podemos incluir coeficientes en fórmulas que no deriven de leyes físicas preexistentes como herramienta modelizadora, y por el otro que podemos "crear" funciones incorporando 
coeficientes o modificando funciones preexistentes. Algunos equipos presenciaron eventos de extinción en su simulador y -aunque no se les encomendó específicamente para hacerloreajustaron parámetros iniciales de sus simuladores (valores de $\mathrm{N}, \mathrm{r}, \mathrm{K}, \gamma$ o $\delta$ ) con el objetivo de estabilizar sus poblaciones hacia un equilibrio, lo que supuso de nuevo una discusión sobre los conceptos de estabilidad, sucesión y equilibrio de un ecosistema.

El análisis con la rúbrica ABPMap, identificó como componentes más desarrolladas las componentes de Conflicto, Contenido, Interdisciplinariedad y Discurso, mientras que las componentes de Apertura y Contexto obtuvieron valores más bajos: Contexto: 2. El proyecto incorpora voces o materiales del mundo real, pero el contexto y rol del alumnado no es verosímil; Conflicto: 4. Los contenidos están ubicados en el núcleo del conflicto a resolver, son esenciales para su resolución y se identifican con él; Discurso: 3. Se aplican dinámicas propias de las ciencias para la obtención de datos y argumentación, pero no se evalúa ni validan procesos de creación del conocimiento científico; Contenidos: 3. Los contenidos son desarrollados de forma parcial y formalizados activamente; Apertura: 1. El alumnado toma algunas decisiones y participa en la evaluación, pero no en la definición de etapas ni objetivos; Interdisciplinariedad: 3. Participan 3 disciplinas STEM.

En lo relativo a los resultados del alumnado en las pruebas de habilidades, la media global de los resultados de las pruebas parece indicar que al terminar la actividad el alumnado muestra mayor nivel de habilidad Tecnológica y Matemática que Científica y los resultados son en general bajos (Fig. 3). Las medias obtenidas en relación a autopercepción parecen indicar que el alumnado en general sobreestima sus habilidades, con una particularidad: de forma general autopercepción de las alumnas es más baja, incluso en el ámbito de la Tecnología, donde la media de resultados de la prueba es más alta que en el grupo de alumnos.
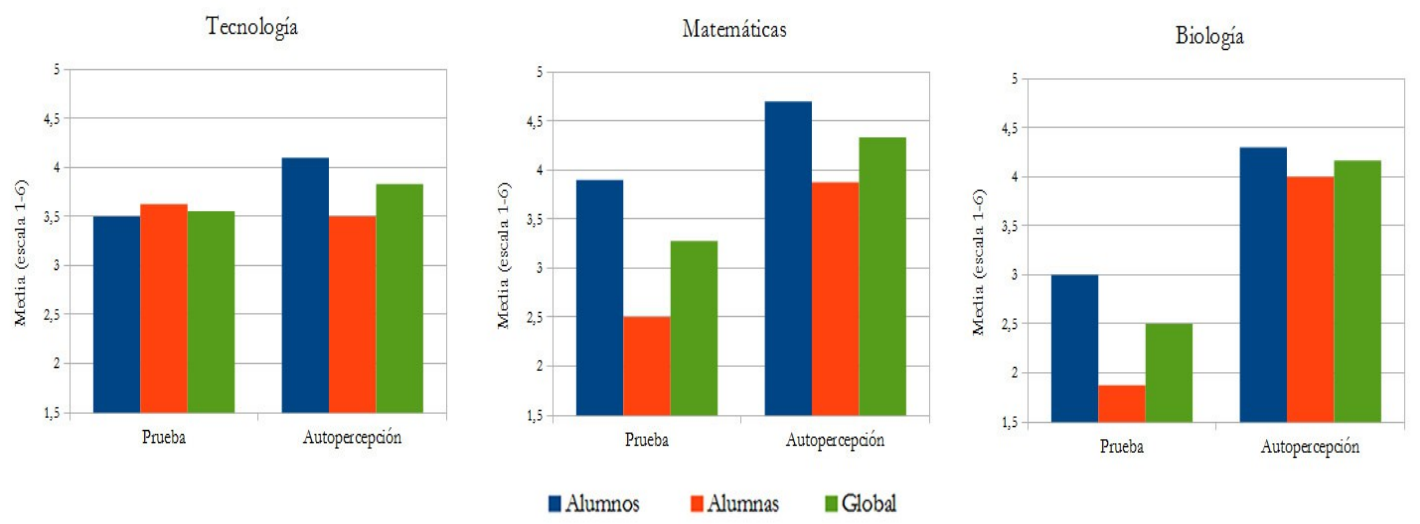

Figura 3. Valores medios globales y separados por sexos, de los resultados de las pruebas de habilidades y la encuesta de auto-percepción. Ambos resultados se cuantifican en una escala del 1 al 6. 
Esta sobreestimación de habilidades no es homogénea (Fig. 4) : en el grupo de las alumnas se aprecian grandes diferencias entre disciplinas, desde una sobreestimación muy elevada en el ámbito de la Biología a lo que ocurre en Tecnología, donde las alumnas subestiman sus habilidades por debajo del resultado real de las pruebas.

No obstante, al analizar con más precisión las posibles diferencias que sugerían las medias separadas por sexos, los resultados del test U Mann Whithney (Tabla 2) indican que las diferencias entre grupos separados por sexos no son significativas, exceptuando los resultados algo más bajos de la habilidad Matemática que obtienen las alumnas en la prueba. Del mismo modo, las distintas medias en relación a la autopercepción no resultan en diferencias significativas entre grupos por sexos.

Tabla 2. Comparación de las medias de resultados de la prueba de habilidades y encuesta de auto-percepción y Prueba U Mann Whitney entre grupos separados por sexos. * : significativo $\operatorname{con} \alpha=0,05$. n.s.: no significativo..

\begin{tabular}{rlcccc}
\hline \multicolumn{1}{l}{ Prueba } & Muestra & Media & $\mathrm{n}$ & $\mathrm{U}$ & $\mathrm{p}$ \\
\hline Tecnología & Alumnos & 3,5 & 10 & 38,5 & 0,928 n.s. \\
Media global: 3,6 & Alumnas & 3,6 & 8 & 17 & $0,045 *$ \\
\hline Matemáticas & Alumnos & 3,9 & 10 & & 0,1 n.s. \\
\hline Media global: 3,3 & Alumnas & 2,5 & 8 & 21 & \\
\hline Biología & Alumnos & 3 & 10 & & \\
\hline
\end{tabular}

\begin{tabular}{rlcccc}
\hline \multicolumn{1}{l}{ Autopercepción } & & & & & \\
\hline Tecnología & Alumnos & 4,1 & 10 & 30 & 0,401 n.s. \\
Media global: 3,8 & Alumnas & 3,5 & 8 & 23 & 0,142 n.s. \\
\hline Matemáticas & Alumnos & 4,7 & 10 & & \\
Media global: 4,3 & Alumnas & 3,9 & 8 & 34,5 & 0,660 n.s. \\
Biología & Alumnos & 4,3 & 10 & &
\end{tabular}

En la encuesta de valoración, los alumnos valoraron de forma muy positiva la interdisciplinariedad: de forma general consideraron que las tres acciones habían resultado útiles en todas las habilidades (valoración en todos los casos por encima de 3,2 de media, independientemente de la área de cada habilidad) (Fig. 5). Con algunos matices. Para el desarrollo del conjunto de habilidades científicas (Biología) (Fig. 5a) el alumnado ha valorado más el trabajo explícito sobre el concepto y en menor grado el trabajo matemático, excepto en 
lo referente a la comprensión del papel de parámetros ecológicos como r, o K, un aprendizaje que parece haberse beneficiado mucho de las aportaciones de Matemáticas y Tecnología (Trabajar fórmulas matemáticas y Programar un simulador) y aparece como el aprendizaje más interdisciplinar con diferencia (acumula 12,8 puntos de las distintas acciones).
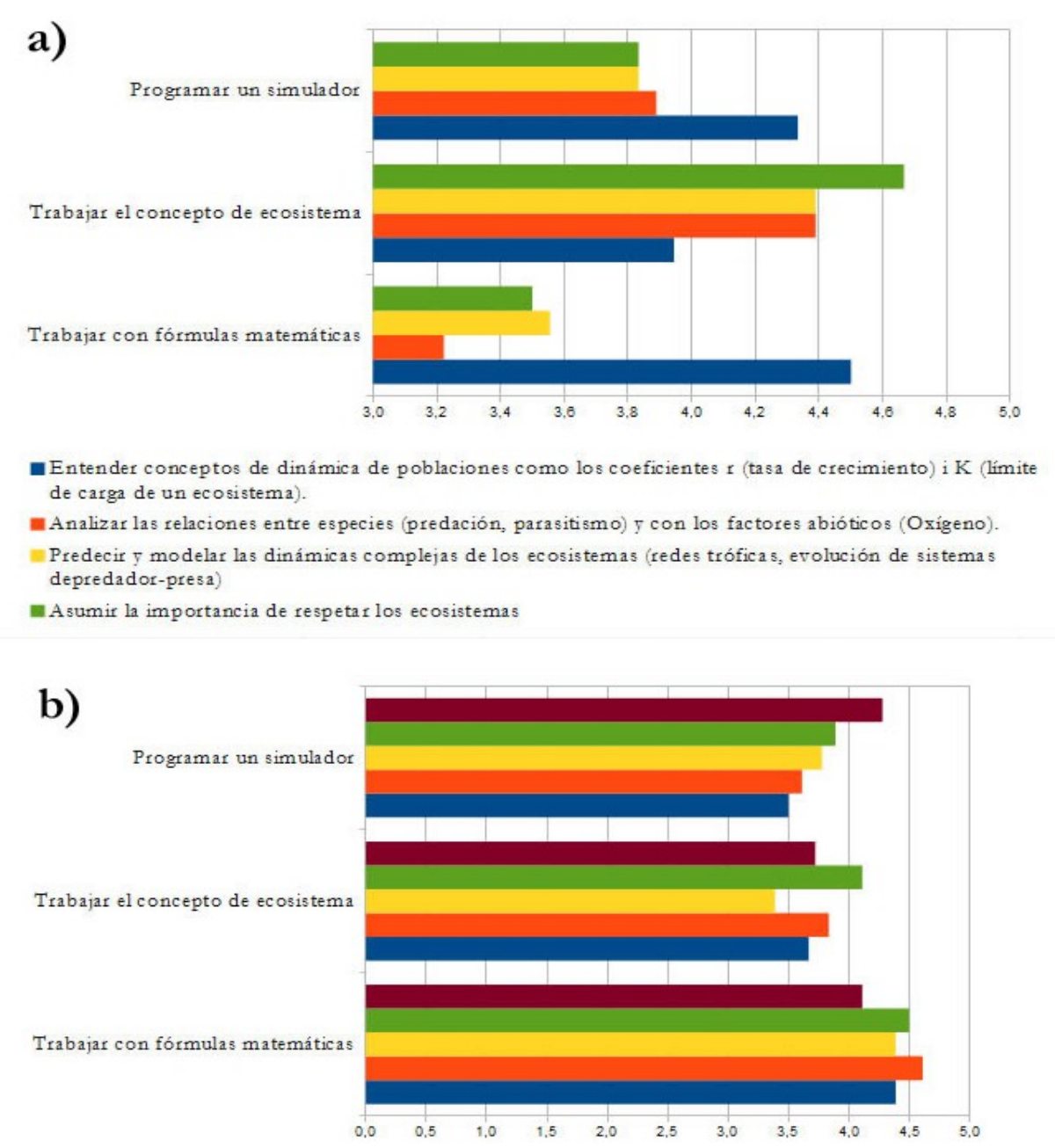

- Comprender el papel matemático de los coeficientes en ecuaciones

= Identificar el comportamiento matemático de las ecuaciones exponencial y logística

Entender el concepto de función y equación matemática

n-Saber usar las matemáticas para representar y analizar sistemas

- Identificar las posibilidades de la programación para resolver problemas

Figura 5. Valoraciones medias (escala $1 \rightarrow 6$ ) realizadas por el alumnado de la utilidad que ha tenido cada enfoque del diseño de la actividad para el desarrollo de distintas habilidades de ámbito científico (a) o Matemático y Tecnológico (b). Notar que para facilitar la comparación entre gráficos, lo ejes se han ajustado al intervalo 3-5.

El desarrollo de las habilidades matemáticas, en especial "Saber usar las matemáticas para representar y analizar sistemas", también recibe aportaciones de forma muy interdisciplinar, dando mucho peso a las acciones del ámbito Científico y Tecnológico (Fig. 5b). En el ámbito tecnológico, representado sólo por una habilidad, la distribución es algo más desigual, recibiendo más impacto de las acciones de tecnología y matemáticas (Programar un simulador y Trabajar el concepto de ecosistema), si bien los valores continúan siendo altos para las tres 
disciplinas. En contraste con todas estas observaciones, al ser preguntados por el impacto del trabajo en grupo (tradicionalmente bien valorado) en el desarrollo de estas habilidades, en todos los casos el impacto se situó en el intervalo $(2,9-3,3)$, un impacto elevado, pero bajo en comparación con los ítems disciplinarios valorados (no mostrado en el gráfico).

\section{Discusión y conclusiones}

El proyecto diseñado ha permitido integrar en una sola actividad el despliegue de contenidos y habilidades de tres ámbitos STEM (Matemáticas, Tecnología y Ciencias) de un modo coherente y vinculando de forma relevante los Contenidos y el Discurso del área a un conflicto o propósito, tal como propone la metodología ABP. El análisis del diseño mediante ABPMap indica que el proyecto descrito tiene una configuración como los "Proyecto de tipo A" definidos en otros trabajos (Domènech-Casal, Lope y Mora 2018). De hecho, la actividad consigue incluir en un mismo propósito (la construcción de un simulador) no sólo contenidos propios de cada área, sino que también sus procesos y miradas propias del área (iteración en Tecnología, modelización en Matemáticas, inferencias y predicciones en relación con modelo ecosistema en Ciencias), una particularidad que se ha comentado con el alumnado de forma explícita a lo largo de la actividad. Consideramos que el carácter tentativo del trabajo del alumnado en la elaboración del simulador (que permite realizar pruebas y tests y confrontarlos con modelos matemáticos y estrategias de programación y relacionarlos con su sentido en los modelos biológicos que describen) es un potente promotor de discusiones "de ida y vuelta" entre los datos y los modelos, en las que se integran y se aprenden de forma conjunta las distintas materias, no meramente un espacio de "aplicación". Muchos alumnos han comprendido el significado de conceptos ecológicos (como K y r) en su uso (tal como propone el ABP) en la construcción del simulador. Es importante en este sentido huir de lo que otros autores han definido como "aplicacionismo" (Cano-Velásquez et al 2018) esto es, pretender que el proyecto sea un mero espacio de aplicación de conceptos que ya se dominan anteriormente en lugar de un espacio de construcción de esos significados. Igualmente, aunque no se ha evaluado explícitamente este aspecto, esas discusiones pueden tener un desarrollo explícito en la secuencia como competencia científica de diseño de experimentos y uso de pruebas. La complejidad de cálculo hace que en algunas ocasiones el fichero Calc se sature y se cierre inadvertidamente. Aunque es un problema, consideramos interesante en Educación Obligatoria utilizar programas que puedan tener aplicaciones no académicas aunque sean menos potentes para la actividad. Además, esto ha conllevado el desarrollo por parte del alumnado estrategias y algoritmos de simplificación de cálculos de interés desde el punto de vista de la programación.

Es importante reseñar desde el punto de vista matemático que la versión de lo que es "modelizar" matemáticamente es incompleta en esta actividad, puesto que explícitamente el alumnado sólo "aplica" o "modifica" tecnológicamente modelos matemáticos preexistentes de lo abstracto a lo concreto, y no "construye" matemáticamente modelos de lo concreto a lo abstracto, aspecto que debería retomarse en alguna otra actividad o como parte previa de esta, por ejemplo "construyendo" el modelo matemático exponencial a partir de experimentos o usando plataformas específicas como GeoGebra. A nivel conceptual la actividad desarrolla poco los factores abióticos (sólo participan el oxígeno y dióxido de carbono) como la temperatura o la radiación solar, por suponer una excesiva complejidad.

Consideramos también que es importante anticipar y tener en cuenta posibles concepciones erróneas que, por necesarias simplificaciones en el diseño, puede desarrollar la actividad, como las siguientes: 
- que todas las especies tienen el mismo tiempo de generación,

- que el crecimiento o cambios en las poblaciones se producen de forma discreta.

- que cada especie participa sólo en un nivel de la red trófica

- que el comportamiento poblacional no depende de fases estacionales o incluso períodos de madurez reproductiva.

El alumnado consigue de media niveles de habilidades Tecnológica y Matemática ligeramente superiores a los que consigue en habilidad Científica. Los resultados obtenidos por el alumnado en las 3 habilidades evaluadas no son excelentes y en el caso de la Biología son incluso bajos, un aspecto que conviene revisar en el diseño de la secuencia y que debe matizar las apreciaciones positivas del alumnado y de forma general en el ámbito de la innovación educativa respecto a la interdisciplinariedad. En contradicción con los resultados, las valoraciones del alumnado apoyan que el trabajo interdisciplinar tiene un impacto positivo en el desarrollo de la idea del ecosistema como espacio dinámico de interacción. Esto parece ser especialmente relevante en la relación entre Matemáticas y Tecnología, pero también entre Matemáticas y Biología, por ejemplo en la comprensión del significado de los conceptos $\mathrm{r}$ y $\mathrm{K}$, el ítem que en opinión del alumnado es desarrollado de forma más interdisciplinar.

Debe tenerse en cuenta que los resultados no se han contrastado con un nivel inicial del alumnado y es difícil descartar además que esto se deba a distintos niveles de dificultad en las preguntas para cada una de las áreas. Por otro lado, las pruebas realizadas son sólo indicativas, al medir sólo una parte de lo enseñado para cada una de las habilidades, y por lo tanto la fiabilidad de estas conclusiones es limitada. En todo caso atribuimos las diferencias aparentes entre disciplinas que probablemente el concepto de Ecosistema ha actuado en esta actividad como "Contexto" en el que desarrollar aprendizajes matemáticos y tecnológicos. Posiblemente, dar más valor científico al contexto (acercándolo al uso del simulador para la resolución de un caso real) podría mejorar el desarrollo de la parte científica de los contenidos de la actividad.

La comparación de las medias de pruebas y autopercepción indica también que el alumnado en general sobreestima sus capacidades en todas las áreas, en especial la Biología. Aunque las medias comparadas por sexos parecen apoyar el sesgo de género en la autopercepción descrito en la bibliografía (UNESCO 2017, Couso 2017) y en trabajos anteriores (Domènech-Casal 2019c), del análisis estadístico de los datos no se extrae que esas diferencias sean significativas, con excepción de los resultados obtenidos en la prueba de matemáticas. Esto quizás se deba al poco tamaño de la muestra y la falta de fiabilidad de los instrumentos.

Como conclusiones a este trabajo, consideramos que:

- El trabajo interdisciplinar desde varias áreas STEM permite realizar aportaciones de cada área al desarrollo de aprendizajes de otras, si bien debe contrastarse su eficacia.

- No se han detectado en este estudio sesgos de género en relación con la autopercepción.

- El alumnado considera útil el trabajo conjunto interdisciplinar con la programación y el trabajo con funciones matemáticas para la comprensión de conceptos clave en ecología como r y K.

Como orientaciones en el diseño de otras actividades STEM, consideramos importante incidir en ofrecer al alumnado herramientas de evaluación que le permitan ajustar su auto-percepción de competencia científica, matemática y tecnológica, más allá de las habilidades propias del área o contexto concreto y modular el papel del Contexto en el diseño de las actividades, de 
modo que permita desarrollar aprendizajes de las distintas áreas participantes de forma equilibrada.

La actividad propuesta se incluye en el itinerario ProyectandoBioGeo (https://sites.google.com/site/proyectandobiogeo/) de despliegue del currículum de Biología y Geología de $4^{\circ}$ de ESO (Domènech-Casal 2017) mediante ABP. El lector encontrará de interés otras experiencias educativas realizadas basadas en la modelización matemática de sistemas ecológicos disponibles en la literatura (Cano-Velásquez et al 2018).

\section{Agradecimientos}

Este artículo ha sido desarrollado en el marco de los proyectos financiados por el Ministerio de Economía y Competitividad con referencias EDU2015-66643-C2-1-P y PGC2018-096581-B-C21 y realizado en el marco del grupo consolidado ACELEC reconocido por la AGAUR (con referencia 2017SGR1399). El autor agradece la colaboración al alumnado participante y el profesorado y direcciones de los centros educativos en los que se ha desarrollado la actividad (Ins Marta Mata, Montornès del Vallès, INS Vilanova, Vilanova del Vallès e INS Marta Estrada, de Granollers) y la discusiones inspiradoras con el equipo coordinador del Programa STEAMCat del Departament d'Educació de la Generalitat de Catalunya y el profesorado de los centros participantes en el programa.

\section{Referencias}

Akerson V.L., Burgess A., Gerber A., Guo M. (2018) Disentangling the meaning of STEM: Implications for Science Education and Science Teacher Education. Journal of Science Teacher Education, 29(1), 1-8. DOI: https://doi.org/10.1080/1046560X.2018.1435063

Bell-Basca B. S., Grotzer T. A., Donis, K. y Shaw S. (2000). Using Domino and Relational Causality to Analyze Ecosystems : Realizing What Goes Around Comes Around The Understandings of Consequence. Project Zero. Harvard Graduate School of Education. National Association of Research in Science Teaching.

Bermúdez G. y De Longhi A. (2008). La Educación Ambiental y la Ecología como ciencia. Revista Electrónica de Enseñanza de las Ciencias, 7(2), 275-297.

Blanco A., España E. y Rodríguez F. (2012) Contexto y enseñanza de la competencia científica. Alambique, Didáctica de las Ciencias Experimentales, 70, 9-18.

Blomhǿj M. (2004) Mathematical modelling - A theory for practice. En Clarke B., Clarke D., Emanuelsson G., Johnansson B., Lambdin D., Lester F., Walby A. y Walby K. (Eds.) International Perspectives on Learning and Teaching Mathematics. National Center for Mathematics Education. Suecia, 145-159.

Brown R., Brown J., Reardon K. y Merrill C. (2011) Understanding STEM: Current perceptions. Technology and Engineering Teacher, 70(6), 5-9.

Bybee R.W. (2010) What is STEM Education? Science, 329 (5995), 996. DOI: $10.1126 /$ science. 1194998

Cano-Velásquez N.A., Zapata-Grajales F.N., Montoya-Osorio J.C. y Villa-Ochoa J.A. (2018). Un juego de rol para el análisis de un modelo matemático en ecología. Revista UNO, Revista de Didáctica de las Matemáticas, 81, 15-21.

Correa C. A. (2013). Estudio de las concepciones alternativas de los alumnos de enseñanza secundaria sobre conceptos estructurantes de ecología a través de la técnica de redes semánticas naturales. Estudios Hemisféricos y Polares, 4, 267-287.

Couso D. (2017) Perquè estem a STEM? Definint l'alfabetització STEM per a tothom i amb valors. Revista Ciències, 34, 21-29. DOI: https://doi.org/10.5565/rev/ciencies.403 
Domènech-Casal J. (2017). Proyectando BioGeo, un itinerario de trabajo por proyectos contextualizados basado en la indagación y la Naturaleza de la Ciencia. Alambique, Didáctica de las Ciencias Experimentales (2017), 89, 54-61.

Domènech-Casal J. (2018). Aprendizaje Basado en Proyectos en el marco STEM. Componentes didácticas para la Competencia Científica. Ápice. Revista de Educación Cientifica, 2(2), 29-42. DOI: https://doi.org/10.17979/arec.2018.2.2.4524

Domènech-Casal J. (2019a). STEM: oportunidades y retos desde la Enseñanza de las Ciencias. Universitas Tarraconensis, monográfico 2019, 155-168. DOI: 10.17345/ute.2019.2.2646

Domènech-Casal J. (2019b). Aprendizaje basado en proyectos, Trabajos prácticos y Controversias. 28 propuestas y reflexiones para enseñar Ciencias. Octaedro: Barcelona.

Domènech-Casal J. (2019c). Packaging, S.L. Un proyecto STEAM sobre Volumen, Área y Diseño. Revista UNO de Didáctica de las Matemáticas, 85, 53-59.

Domènech-Casal J., Lope S. y Mora L. (2019). Qué proyectos STEM diseña y qué dificultades expresa el profesorado de secundaria sobre Aprendizaje Basado en Proyectos. Revista Eureka sobre Enseñanza y Divulgación de las Ciencias 16(2), 2203. DOI: https://doi.org/10.25267/Rev_Eureka_ensen_divulg_cienc.2019.v16.i2.2203

Duranti A. y Goodwin C. (Eds). (1992) Rethinking context: Language as an interactive phenomenon. Cambridge: Cambridge, University Press.

Eilam B. (2002). Strata of Comprehending Ecology: Looking Through the Prism of Feeding Relations. Science Education, 86(5), 645-671. https://doi.org/10.1002/sce.10041

García J.E. (1997). La formulación de hipótesis de progresión para la construcción del conocimiento escolar : una propuesta de secuenciación en la enseñanza de la ecología. Alambique, Didáctica de las Ciencias Experimentales 14, 37-48. http://hdl.handle.net/11162/25265

García J.E. (2003). Investigando el ecosistema. Investigación en la escuela, 51, 83-100. DOI: http://dx.doi.org/10.12795/IE.2003.151.07

Gil M. J. y Martinez B. (1992). Problemática en la enseñanza/aprendizaje de la ecología. Revista Interuniversitaria de Formación del Profesorado, 14, 67-70.

Gilbert J.K. (2006) On the nature of context in chemical education. International Journal of Science Education, 28(9), 957-976. DOI: https://doi.org/10.1080/09500690600702470

Grotzer T. A. y Bell Basca B. (2003). How does grasping the underlying causal structures of ecosystems impact students' understanding? Journal of Biological Education, 38(1), 16-29. https://doi.org/10.1080/00219266.2003.9655891

Jaén M., Esteve P. y Baños I. (2018). Problemáticas ambientales en las que confluyen ciclos biogeoquímicos. Propuesta para la educación secundaria. Apice. Revista de Educación Cientifica, 2(1), 30-39. DOI: https://doi.org/10.17979/arec.2018.2.1.3162

Kilpatrick W.E. (1918). The Project Method: the use of the purposeful act in the educative process. New York: Teachers college, Columbia University.

Larmer J., Mergendoller J. y Boss S. (2015) Setting the Standard for Project Based Learning: A Proven Approach to Rigorous Classroom Instruction. ASCD, Alexandria.

Leach J., Driver R., Scott P. y Wood-Robinson C. (1996a). Children's ideas about ecology 2: ideas found in children aged 5-16 about the cycling of matter. International Journal of Science Education, 18(1), 19-34. https://doi.org/10.1080/0950069960180102 
Leach J., Driver R., Scott P. y Wood-Robinson C. (1996b). Children's ideas about ecology 3: Ideas found in children aged 5-16 about the interdependency of organisms. International Journal of Science Education, 18(2), 129-141. https://doi.org/10.1080/0950069960180201

Maldonado F., González F. y Jiménez M.P. (2007). Las ilustraciones de los ciclos biogeoquímicos del carbono y el nitrógeno en los textos de secundaria. Revista Eureka de Enseñanza y Divulgación de las Ciencias, 4(3), 442-460. https://revistas.uca.es/index.php/eureka/article/view/3786

Margalef R. (1981). Ecología. Barcelona. Editorial Planeta.

Martín-Páez T., Aguilera D., Perales-Palacios F.J. y Vilchez-González J.M. (2019). What are we talking about when we talk about STEM education? A review of literature. Science Education, 2019, 1-24. https://doi.org/10.1002/sce.21522

Mohan L., Chen J. y Anderson C. W. (2009). Developing a multi-year learning progression for carbon cycling in socio-ecological systems. Journal of Research in Science Teaching, 46(6), 675-698. DOI: http://10.1002/tea.20314

Murillo J. y Gil M.J. (2009). Uso del concepto de sucesion ecólogica por alumnos de secundaria: La prediccion de los cambios en los ecosistemas. Enseñanza de las Ciencias, $27(1)$, 19-32. https://www.raco.cat/index.php/ensenanza/article/viewFile/132204/332988

Rocard M., Csermely P., Jorde D., Lenzen D., Walwerg-Heriksson H. y Hemmo V. (2007) Science Education Now: a new pedagogy for the future of Europe. Report for the European Comission. http://ec.europa.eu/research/sciencesociety/document_library/pdf_06/report-rocard-on-science-education_en.pdf

Rojero F. F. (1999). Entender la organización: aspectos didácticos del estudio de los ecosistemas. Alambique: Didáctica de las ciencias experimentales, (20), 55-64.

Sainz M, Castaño C., Meneses J., Fàbregues S., Müller J., Rodó M., Martínez J.L., Romano M.J., Arroyo L. y Garrido N. (2017). ¿Por qué no hay más mujeres STEM? Se buscan ingenieras, físicas y tecnólogas. Madrid: Ariel.

Sánchez F. J. y Pontes A. (2010). La comprensión de conceptos de Ecología y sus implicaciones para la educación ambiental. Revista Eureka Sobre Enseñanza y Divulgación de Las Ciencias, 7, 271-285. http://hdl.handle.net/10498/8942

Sanmartí N. y Márquez C. (2017) Aprendizaje de las ciencias basado en proyectos: del contexto a la acción. Ápice, Revista de Educación Científica, 1(1), 3-16. DOI: https://doi.org/10.17979/arec.2017.1.1.2020

Shepardson D. P., Wee B., Priddy M. y Harbor J. (2007). Students' mental models of the environment. Journal of Research in Science Teaching, 44(2), 327-348. DOI: http://10.1002/tea.20161

Simarro C. y Couso D. (2016) Análisis de una actividad tinkering en el marco de la educación STEM. Comunicación y Pedagogía 291-292, 65-7.

Smith R.L. y Smith T.M. (2001). Ecología. Madrid: Addison Wesley.

UNESCO (2017). Cracking the code: girls' and women's education in science, technology, engineering and mathematics (STEM). UNESCO, Paris.

Vilches A. y Gil D. (2007). Emergencia planetaria: necesidad de un planteamiento global. Educatio siglo XXI: Revista de la Facultad de Educación, 7 (25), 19 -50. 
White P. A. (2000). Naive analysis of food web dynamics: A study of causal judgment about complex physical systems. Cognitive Science, 24(4), 605-650.
https://doi.org/10.1207/s15516709 $\operatorname{cog} 2404 \_3$

Wing J.M. (2006). Computational Thinking. Communications of the ACM, 49(3), 33-35. 